\title{
Molecular basis of oculocutaneous albinism type 1 in Lebanese patients
}

Received: 15 March 2005/ Accepted: 25 April 2005/Published online: 4 June 2005

(C) The Japan Society of Human Genetics and Springer-Verlag 2005

\begin{abstract}
Oculocutaneous albinism type 1 (OCA1) results from mutations in the tyrosinase gene, which lead to partial or complete loss of activity of the corresponding enzyme. A large number of mutations have been identified worldwide, providing insight into the pathogenesis of the disorder. We performed ophthalmic and dermatological exams on 30 Lebanese subjects with oculocutaneous albinism, then screened for mutations in the tyrosinase gene in an effort to establish the molecular basis of the disorder in our population and correlate it with phenotypic findings. The five exons of the gene together with the exon-intron boundaries and part of the promoter region were sequenced. Mutations were found in a total of 14 patients $(47 \%)$ while no mutation was identified in the sequenced regions in $53 \%$ of patients. Fourteen different mutations were identified of which eight were novel while six had been previously reported. Mutations were mainly seen in patients with clinical findings, suggestive of OCA1A (64\% of patients with OCA1A versus $25 \%$ of patients with OCA1B); therefore, the absence of mutations in some of the other patients may indicate the involvement of other genes.
\end{abstract}

\footnotetext{
L. Zahed $(\bowtie) \cdot H$. Zahreddine

Department of Pathology and Laboratory Medicine,

American University of Beirut Medical Center,

Beirut, Lebanon

E-mail: lailaz@aub.edu.lb

Tel.: +961-1-350000

Fax: +961-1-370845

B. Noureddine

Department of Ophthalmology,

American University of Beirut Medical Center,

Beirut, Lebanon

N. Rebeiz $\cdot$ F. Haddad

Department of Dermatology,

American University of Beirut Medical Center,

Beirut, Lebanon

N. Shakar · P. Zalloua

Genetics Laboratory, Chronic Care Center,

Hazmieh, Lebanon
}

Keywords OCA1 - Tyrosinase gene mutations ·

Lebanon

\section{Introduction}

Oculocutaneous albinism (OCA) is manifested by reduced synthesis of melanin, which may result from mutations in a variety of genes (Goto et al. 2004; Opitz et al. 2004; King et al. 2003). As the name indicates, in OCA, hypopigmentation is both cutaneous and ocular, and in the most common types of OCA, other tissues and organs are not significantly involved. Hypopigmentation of the skin is associated with psychological and social problems, decreases natural protection against sunburn, and results in predisposition to skin cancer. In the absence of melanin in the eye, development of the visual pathways is abnormal, resulting in decreased visual acuity, strabismus, nystagmus, and photophobia.

One of the most common types of OCA is OCA1 (MIM\#203100), an autosomal recessive disorder of reduced pigmentation in the hair, skin, and eyes, resulting from mutations in the tyrosinase gene (TYR) (MIM\#606933), a five-exon gene spanning approximately $60 \mathrm{~kb}$ on chromosome 11q. Two different subtypes of OCA1, A and B, can be distinguished in humans based mainly on clinical observations (King et al. 2003). The OCA1A is the classic, more severe form of the disorder, in which tyrosinase activity is completely deficient. Consequently, the skin and hair are white throughout life, eyes are blue with translucent irides, visual acuity is greatly decreased, and other ocular problems are severe. In OCA1B, there is minimal tyrosinase activity due to milder mutations, resulting in little or no melanin pigmentation at birth; however, gradual pigmentation of skin and hair may occur to a variable degree, and ocular problems are generally less severe. This clinical classification of OCA may sometimes be misleading, as phenotypes resulting from 
mutations in other genes may overlap with the milder TYR mutations (King et al. 2003). The current recommendation is therefore to base the clinical characterization of OCA on the type of gene involved (King et al. 2003).

The molecular basis of OCA1 is quite heterogeneous, and more than 190 mutations in the TYR gene have been identified so far in different population groups (Albinism Database; Opitz et al. 2004). The identification of mutations is important to confirm the diagnosis of OCA1, to understand the function of the tyrosinase protein, to elucidate the basis of the disorder in specific populations, and to provide carrier testing and genetic counseling, when requested. In this study, we performed ophthalmic and dermatological exams on 30 unrelated Lebanese subjects with oculocutaneous albinism, then screened for mutations in the tyrosinase gene in an effort to establish the molecular basis of the disorder in our population and correlate it with phenotypic findings.

\section{Subjects and methods}

\section{Subjects}

Thirty patients with OCA were referred either through the ophthalmology or dermatology clinic, or because they, or one of their acquaintances, had heard about the study and were interested in participating. When possible, thorough ophthalmic and dermatologic exams were performed by some of the authors. Otherwise, results of the most recent ophthalmic exam were requested, and pigment history was obtained. A clinical information form was filled out by the major author, including this data, in addition to family history, pedigree, religion, and geographical origin of each patient. Since detailed ophthalmic and/or dermatologic data could not be obtained on some of the patients, the clinical criteria to classify patients as OCA1A were the presence of white hair and skin throughout life (King et al. 2003). The group of OCA1B patients included those who initially had white scalp hair at birth but developed pigment in the first decade of life, and a group of uncertain OCA1B classification who had some pigmentation at presentation but an unclear pigment history (King et al. 2003).

\section{Methods}

After informed consent from all patients, DNA was obtained from peripheral blood lymphocytes by salt extraction. Seven different fragments from the TYR gene covering $500 \mathrm{bp}$ of the promoter region, each exon and exon-intron boundaries, as well as part of the adjacent introns, were PCR-amplified. The sequences of the primers used were kindly provided by the Genetics Laboratory at the International Albinism Center. Amplified fragments were sequenced using the Thermo Sequenase II dye terminator cycle kit (Amersham, OH,
USA) and run on an ABI 310 fluorescent sequencer (Applied Biosystems, CA, USA). Some of the fragments were commercially sequenced (AGOWA, Germany) following PCR amplification.

\section{Results}

Of the 30 recruited patients, $14(47 \%)$ had clinical features suggestive of OCA1A, with white skin and hair since birth, while the rest of the patients had some pigmentation at presentation and therefore suggestive of OCA1B (11 patients, 37\%) or another type of OCA (five patients, $16 \%$ ). Consanguinity (mainly third degree) was noted in the parents of 22 of the patients studied $(73 \%)$, indicating that consanguinity plays an important role in the frequency of the disorder in our country, as previously observed for other hereditary disorders (Zahed and Bou-Dames 1997; Zahed et al. 1999; Nabulsi et al. 2003). In $21(70 \%)$ families, there was more than one affected individual, and in eight of these (38\% of total), affected individuals were found in two different generations.

Mutations were identified in a total of 14 patients $(47 \%)$. Nine $(64 \%)$ of these patients had been characterized as OCA1A while five $(36 \%)$ had been clinically classified as OCA1B. Thus, a mutation was identified in the TYR gene in $64 \%$ of OCA1A patients, in $45 \%$ of the OCA1B patients, and in none of the five patients with the uncertain OCA1B classification.

Due to the high frequency of consanguinity, the majority of patients with identifiable mutations $(71 \%)$ were homozygous for one mutation while only $10 \%$ were compound heterozygotes. A single mutation was identified in one patient only.

In total, 14 different mutations (Table 1) were identified, four of which were seen in two unrelated patients each. Five mutations were already included in the

Table 1 Tyrosinase (TYR) gene mutations identified in this study, including number of patients seen with each mutation and clinical presentation as oculocutaneous albinism (OCA) 1A or B

\begin{tabular}{ll}
\hline Mutation & $\begin{array}{l}\text { Number } \\
\text { of patients }\end{array}$
\end{tabular}

\begin{tabular}{llll}
\hline cd50 C $>$ A Ser $>$ Stop & 1 & B & Christian Arab \\
cd84 T $>$ G Tyr $>$ Stop & 1 & A & Novel \\
cd256 C $>$ T His $>$ Tyr & 2 & B & Caucasian \\
cd272 T $>$ C Trp $>$ Arg & 2 & A & Novel \\
cd299 G $>$ A Arg $>$ His & 1 & A & Christian Arab \\
cd342 T $>$ C Asn $>$ Asn & 1 & A & Novel \\
del 15 bp IVS2 & 1 & A & Novel \\
cd346 G $>$ A Gly $>$ Glu & 1 & A & Novel \\
cd359 A $>$ T Gln $>$ Leu & 1 & A & Novel \\
cd360 A $>$ G Ser $>$ Gly & 1 & B & Novel \\
del A cd402 & 1 & B & Novel \\
cd402 C $>$ T Arg $>$ Stop & 2 & A & Christian Arab \\
cd433 A $>$ G Tyr-Cys & 1 & B & Novel \\
cd448 G $>$ A Asp $>$ Arg & 2 & A & Caucasian \\
\hline
\end{tabular}

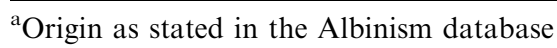


Albinism Database while nine were novel and, to our knowledge, have not been previously reported. The mutation at codon $50(\mathrm{C}>\mathrm{A})$, previously reported in individuals of Christian Arab origin, was found in a Muslim patient while the $\mathrm{G}>\mathrm{A}$ mutation at codon 299 and the $\mathrm{C}>\mathrm{T}$ mutation at codon 402 were found in Christian patients, as previously reported (Albinism Database). The other two mutations ( $\mathrm{cd} 256 \mathrm{C}>\mathrm{T}$ and cd448 G>A), reportedly of Caucasian origin, were found in two unrelated patients each.

Among the nine new mutations identified, one was a nonsense mutation (cd84 $\mathrm{T}>\mathrm{G}$ ) while five were missense mutations, including the $\mathrm{T}>\mathrm{C}$ at codon 272 seen in two unrelated patients. One frameshift mutation (deletion of $\mathrm{A}$ at cd402) was detected. The $\mathrm{T}>\mathrm{C}$ mutation at codon 342 does not result in an amino acid change in the final protein product and may therefore represent a polymorphism. The same thing may be true for the 15 base pair deletion in IVS2, more than 60 bases upstream from the exon three splice-site junction. This deletion was noted in an individual with two other point mutations (cd84 $\mathrm{T}>\mathrm{G}$ and cd346 $\mathrm{G}>\mathrm{A}$ ), each of which might explain the clinical presentation. This individual was thus homozygous for three different mutations, at least two of which are pathogenetic. The accumulation of three to five mutations in the tyrosinase gene has been previously reported (Passmore et al. 1999), and more recently, Opitz et al. (2004) reported it in $20 \%$ of the patients included in their study.

Mutations were detected in all exons of the TYR gene with the exception of exon 5, where reported mutations are less frequent (Albinism Database). The association of the previously reported mutations with the OCA1A or B subtype was different in some of the patients included in our study (Table 1) when compared with the association reported in the Albinism Database. Indeed, patients with a homozygous mutation at $\operatorname{cd} 50(\mathrm{C}>\mathrm{A})$ or at cd256 $(\mathrm{C}>\mathrm{T})$ were clinically classified as OCA1B while the reported phenotype on the database is OCA1A. Similarly, the adult patient homozygous for the mutation at cd448 $(\mathrm{G}>\mathrm{A})$ presented as OCA1A whereas this mutation was previously reported to result in the milder B subtype. These observations may be explained by the fact that all our patients were homozygous for these TYR alleles due to parental consanguinity.

\section{Discussion}

In this study - in which sequencing of all exons of the TYR gene, the exon-intron boundaries, and the promoter region were performed - mutations were identified in only $14(47 \%)$ of the patients included. Several reasons may explain the lack of identifiable mutations in some patients. In patients suggestive of OCA1 and involvement of the TYR gene, this may be due to the fact that a mutation may have been present in a region of the gene that was not sequenced by our methodology, such as a regulatory sequence or an intron. On the other hand, and especially in patients of uncertain clinical classification, involvement of other genes, such as the $\mathrm{P}$ gene, must be suspected. Mutations in the $\mathrm{P}$ gene have been identified in some patients who had white hair at birth (King et al. 2003; Passmore et al. 1999), indicating a clinical overlap between patients with different types of OCA.

\section{References}

Goto M, Sato-Matsumura KC, Sawamura D, Yokota K, Nakamura H, Shimizu H (2004) Tyrosinase gene analysis in Japanese patients with oculocutaneous albinism. J Dermatol Sci 35:215-220

King RA, Pietsch J, Fryer JP, Savage S, Brott MJ, Russell-Eggitt I, Summers CG, Oetting WS (2003) Tyrosinase gene mutations in oculocutaneous albinism 1 (OCA1): definition of the phenotype. Hum Genet 113:502-513

Mutations in the tyrosinase gene associated with OCA1, Albinism Database, http://albinismdb.med.umn.edu/oca1mut.html

Opitz S, Kasmann-Kellner B, Kaufmann M, Schwinger E, Zuhlke C (2004) Detection of 53 novel DNA variations within the tyrosinase gene and accumulation of mutations in 17 patients with Albinism. Hum Mutat 23:630-631

Nabulsi MM, Tamim H, Sabbagh M, Obeid MY, Yunis KA, Bitar FF (2003) Parental consanguinity and congenital heart malformations in a developing country. Am $\mathrm{J}$ Med Genet 116A:342-347

Passmore LA, Kaesmann-Kellner B, Weber BH (1999) Novel and recurrent mutations in the tyrosinase gene and the $\mathrm{P}$ gene in the German albino population. Hum Genet 105:200-210

Zahed L, Nabulsi M, Bou-Ghanim M, Usta I (1999) Acceptance of prenatal diagnosis for genetic disorders in Lebanon. Prenat Diagn 19:1109-1112

Zahed L, Bou-Dames J (1997) Acceptance of first-trimester prenatal diagnosis for the haemoglobinopathies in Lebanon. Prenat Diagn 17:423-428 